\title{
Selective Dichroic Patterning by Nanosecond Laser Treatment of Ag Nanostripes
}

\author{
Juan R. Sanchez-Valencia, Johann Toudert, * Ana Borras, * Angel Barranco, Ruth Lahoz, \\ German F. de la Fuente, Fabian Frutos, and Agustin R. Gonzalez-Elipe
}

The strong absorption of light ${ }^{[1]}$ and the local amplification of the electromagnetic field ${ }^{[2]}$ at the plasmon resonance of noble metal nanostructures have been the focus of hundreds of studies due to their practical applications for the fabrication of optical devices such as filters, non-linear optical components, or Raman enhancers. ${ }^{[3,4]}$ The control of the plasmon features such as spectral width, ${ }^{[5]}$ position, ${ }^{[6]}$ and shape ${ }^{[7]}$ can be accomplished by different physical deposition routes ${ }^{[8-12]}$ providing adequate growing conditions of metal nanoparticles (MNPs). Pioneer works in the 1990s showed the optical selectivity of elongated $\mathrm{Ag}$ deposits on $\mathrm{SiO}_{2}$ with applications as optical filters for windows to control solar heat gain and glare, among others. ${ }^{[13]}$ Recently, assemblies of parallel stripes of MNPs have been fabricated onto preformed surfaces presenting a 1D periodic roughness ${ }^{[14,15]}$ or bundled $\mathrm{SiO}_{2}$ nanocolumns..$^{[16,17]} \mathrm{A}$ significant macroscopic optical dichroism has been reported for these systems that can be useful for the development of polarized light emitters or materials with an enhanced IR luminescence because of the excitation of two distinct plasmon resonances in the directions parallel (longitudinal mode) and perpendicular (transverse mode) to the stripes. ${ }^{[18]}$ Architecture control of the metal assemblies plays a determinant role in the functional properties of the material. For this purpose, the softlithographic techniques provide means to accurately tailor the nanostructure of the materials. ${ }^{[18-21]}$ Laser scanning is a softlithographic technique widely used to modify the shape and structure of metal nanoparticles. ${ }^{[21-24]}$ Surface modification can be easily achieved by in situ ${ }^{[21]}$ or ex situ ${ }^{[22-24]}$ pulsed laser treatment in the case of random systems of MNPs. In contrast, nothing has been reported about the effect of a pulsed laser on the structure and optical dichroism of autoorganized metal

Dr. J. R. Sanchez-Valencia, Dr. J. Toudert, Dr. A. Borras,

Dr. A. Barranco, Prof. Dr. A. R. Gonzalez-Elipe

Nanotechnology on Surfaces Laboratory

ICMSE: Materials Science Institute of Seville

CSIC-University of Seville

Avd. Americo Vespucio 49, 41092, Sevilla, Spain

E-mail: johann.toudert@gmail.com; anaisabel.borras@icmse.csic.es

Dr. R. Lahoz, Prof. G. F. de la Fuente

Materials Science Institute of Aragon

CSIC-University of Zaragoza

C/María de Luna 3, 50018, Zaragoza, Spain

Dr. F. Frutos

Applied Physics Department

E.T.S. Ingeniería Informática

University of Seville

Avd. Reina Mercedes s/n, 41012 Seville, Spain nanostructures. In this paper we show that nanosecond (ns) laser irradiation can be effectively used to control the optical dichroism of Ag stripes supported on $\mathrm{SiO}_{2}$ nanocolumns (NCs). This dichroism can be effectively tailored along the full visible range. Thus, we propose the utilization of the $\mathrm{AgNPs} / \mathrm{SiO}_{2} \mathrm{NCs}$ structures for writing dichroic patterns at the microscale with potential applications for encryption and data storage purposes.

$\mathrm{AgNPs} / \mathrm{SiO}_{2} \mathrm{NCs}$ films were grown by a two-step process. ${ }^{[17]}$ First, $\mathrm{SiO}_{2}$ thin films were deposited by glancing angle vapor deposition (GLAD) with a tilted columnar nanostructure and $\approx 350 \mathrm{~nm}$ thickness (see Figure S1a in the Supporting Information and the Experimental Section). ${ }^{[25,26]}$ These structures present an anisotropic surface topography known as "bundling", ${ }^{[17]}$ consisting of the coalescence of the NCs along the $x$-direction (Figure S1b). The silver nanoparticles were then grown by DC sputtering at room temperature. The "bundled" $\mathrm{SiO}_{2} \mathrm{NCs}$ act as a template for the fabrication of Ag stripes formed by metal NPs. ${ }^{[17]}$ Nanosecond laser post-treatment was then performed at normal incidence. The energy impinging on the sample surface was controlled by varying the output laser power $(P)$ between $3.5 \mathrm{~kW} \mathrm{~cm}^{-2}$ and $7.0 \mathrm{~kW} \mathrm{~cm}^{-2}$.

Figure 1 shows the scanning electron microscopy (SEM) images of a film before (Figure 1a,c,e) and after (Figure 1b,d,f) laser treatment at $7.0 \mathrm{~kW} \mathrm{~cm}{ }^{-2}$. The surface of the original film consists of stripes of densely packed MNPs following the bundling direction of the nanocolumns ( $x$-direction) (Figure 1a). ${ }^{[17]}$ These Ag-striped nanostructures, separated from each other around $30 \mathrm{~nm}$ in the $y$-direction, cover $78 \%$ fraction of the surface (defined as the portion of the image corresponding to MNPs) as obtained by digital picture analysis. Figure 1c,e show that the particles present a "carpet-like" projected shape with a height $\approx 15-20 \mathrm{~nm}$. After laser treatment at $7.0 \mathrm{~kW} \mathrm{~cm}{ }^{-2}$ (Figure $1 \mathrm{~b}, \mathrm{~d}, \mathrm{f}$ ), the parallel stripes are still present but are formed by continuous and extremely smooth metal islands (see also atomic force microscopy (AFM) image in Figure 2). In addition, these islands appear to be more separated along the $y$-direction. Indeed, while their center-to-center distance remains unchanged, their border-to-border distance has increased to $50 \mathrm{~nm}$, with a surface coverage of only $51 \%$. The laser-treated metal stripes present a truncated ellipsoidal projected shape in the $y-z$ plane (Figure $1 \mathrm{~d}, \mathrm{f}$ ). Their estimated height of $\approx 30-40 \mathrm{~nm}$ is higher than before laser treatment. Furthermore, separated nanoparticles in the 10-20 $\mathrm{nm}$ range appear on the lateral faces of the $\mathrm{SiO}_{2}$ columns (Figure 1e,f and Figure S2 in the Supporting Information) in addition to the metal stripes. Figure 2 shows the AFM topography pictures of the as-grown sample (a) and the laser-treated samples at laser power of $4.0 \mathrm{~kW} \mathrm{~cm}^{-2}$ (b) and $7.0 \mathrm{~kW} \mathrm{~cm}{ }^{-2}$ (c). The surface of the original sample 


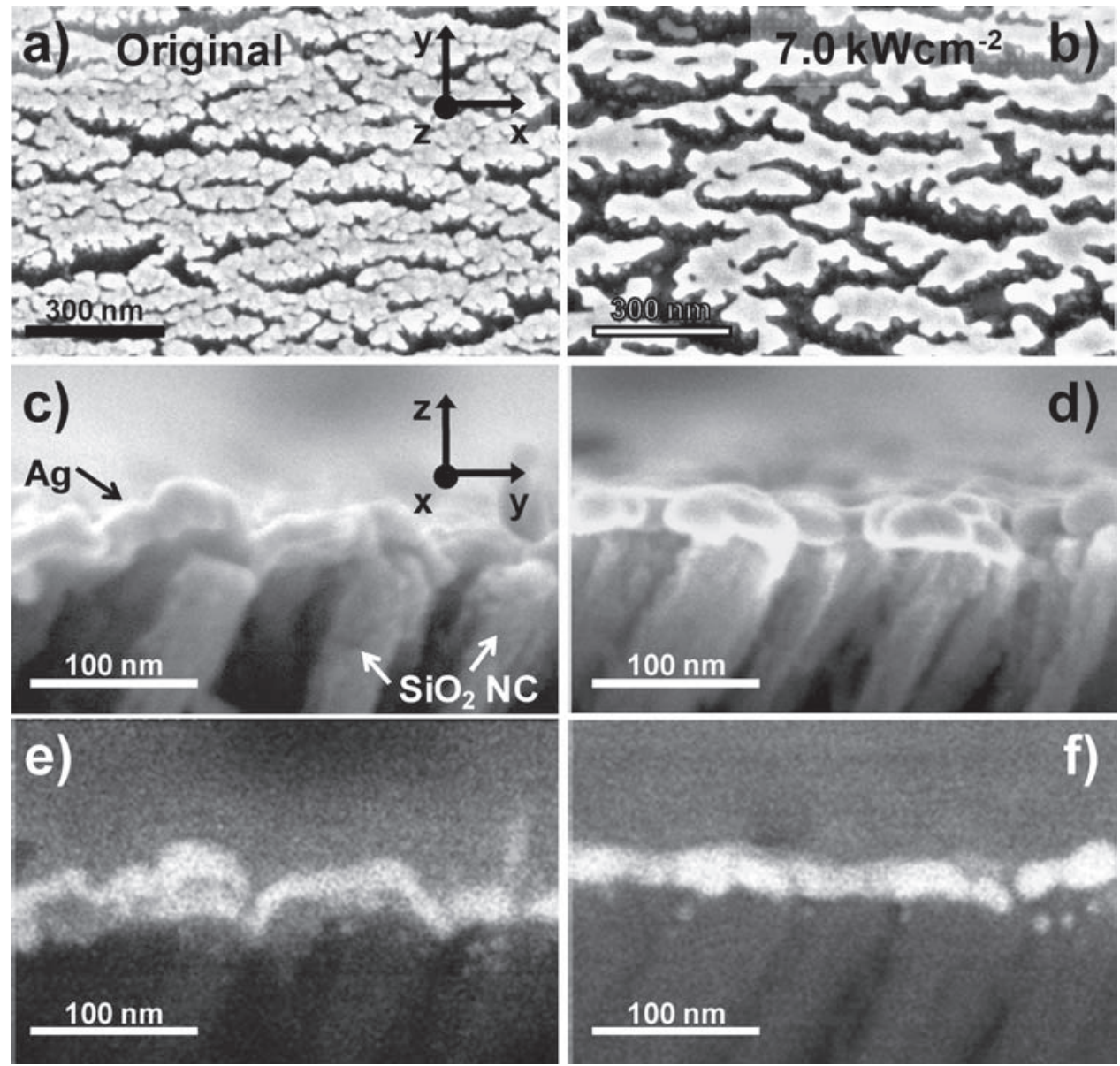

Figure 1. Surface $(a, b)$ and cross-section (c,d) SEM images of $\mathrm{AgNPs} / \mathrm{SiO}_{2} \mathrm{NCs}$ films before (left) and after (right) laser treatment at $7.0 \mathrm{~kW} \mathrm{~cm}^{-2}$; e) and f) correspond to the backscattered electron images of (c) and (d), respectively.

(Figure 2a) is formed by densely packed nanoparticles, in agreement with the SEM normal view microscopy image in Figure 1a. The analysis of the AFM images shows that these Ag NPs have a wide size distribution ranging from 20 to $100 \mathrm{~nm}$. Such a high dispersion in the Ag NPs sizes is known to produce a wide plasmon absorption band along the visible range. ${ }^{[1-3]}$ The AFM topography pictures of the laser treated sample at $4.0 \mathrm{~kW} \mathrm{~cm}^{-2}$ does not reveal a substantial change in the size distribution of the Ag NPs although the particle edges are now less defined. This indicates that the MNPs have begun to percolate. Such a percolation can be easily deduced from the derivative representation of the AFM topographic images shown in Figure 2e. This representation enhances the contrast at the particle edges while the regions of a constant slope are shown with the same color. Accordingly, in the original samples the Ag NPs present very well defined borders, while in the sample treated at $4.0 \mathrm{~kW} \mathrm{~cm}^{-2}$ laser power the edges are less defined. The most outstanding topographic change occurs for the laser treated sample at $7.0 \mathrm{~kW} \mathrm{~cm}^{-2}$ (Figure 2c,f). For this treatment the Ag NPs are completely percolated in very smooth and continuous nanostripes in the $x$-direction. Figure $2 \mathrm{~g}$ represents the statistical analysis of the grain borders of the features in the surface obtained for the three modified surfaces in Figure $2 \mathrm{~d}-\mathrm{f}$. The results can also be interpreted as the comparison of the size distributions of the metal grains in the surfaces, showing that the laser treatment induces a smoothing in the metal nanoparticles surface as well as a sharpening in the size distribution of the features in the surface consistent with the enlongation of the Ag nanostripes in the $x$-direction. Moreover, it is also worth mentioning that the analysis of the AFM microscopy images by fast Fourier transform in two dimensions (2DFFT) (see Experimental Section for further details) shows an increase of the anisotropy after treatment at high laser power. Figure 3 (left), which shows the SEM images of the samples, confirms that the $\mathrm{Ag}$ nanostructures evolve as a function of the laser power (see also Figure S3, Supporting Information). The comparison between Figure 3a (asgrown material) and Figure $3 \mathrm{~b}$ $\left(P=4.0 \mathrm{~kW} \mathrm{~cm}^{-2}\right)$ confirms the result observed by AFM that continuous and smooth metal stripes already form at intermediate powers. Further increase in the power (Figure 3c; $P=$ $7.0 \mathrm{~kW} \mathrm{~cm}^{-2}$ ) produces a decrease in the metal coverage that is likely to be linked to an increase of the stripe height.

The consequences of the structural changes provoked by the laser treatment on the optical response of the films were studied by transmittance measurements at normal incidence with a collimated and linearly polarized beam. Spectra in Figure 3d were acquired with the incoming electric field oriented along the stripes ( $x$-polarization) and those in Figure $3 e$ with the field perpendicular to the stripes ( $\gamma$-polarization) of $\mathrm{AgNPs} / \mathrm{SiO}_{2} \mathrm{NCs}$ films before and after treatment at different laser output powers. The spectrum of the original sample presents absorption bands peaking at $650 \mathrm{~nm}$ and $550 \mathrm{~nm}$ for $x$ - and $y$ polarization, respectively. This optical dichroism must result from the excitation of distinct plasmon resonances along the stripes (longitudinal plasmon) and perpendicularly to them (transverse plasmon), while the shift between the two bands indicates that the electromagnetic coupling along the stripes is stronger than between successive stripes.

Upon laser treatment at moderate power $\left(P=4.0 \mathrm{~kW} \mathrm{~cm}{ }^{-2}\right)$, the longitudinal plasmon band (Figure $3 \mathrm{~d}$ ) becomes less intense, broadens, and shifts towards the infrared. These features are typical of the formation of continuous metal stripes in agreement with Figure 3b. Increasing the laser power has a smaller effect on this broad band, which becomes progressively 

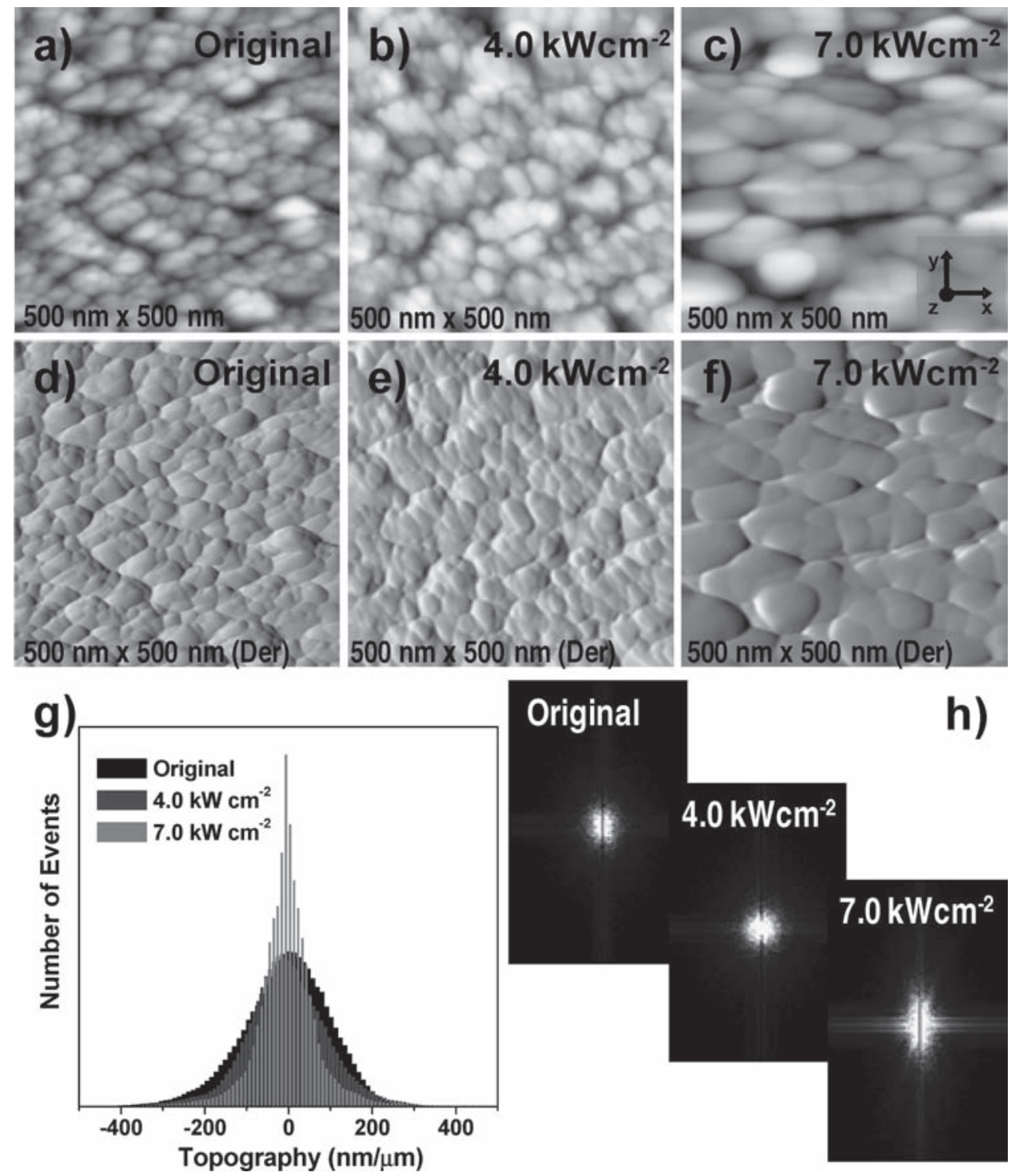

Figure 2. AFM microscopy images of the $\mathrm{AgNPs} / \mathrm{SiO}_{2} \mathrm{NCs}$ thin films before the laser treatment (a), after treatment at $P=4.0 \mathrm{~kW} \mathrm{~cm}^{-2}$ (b) and at $P=7.0 \mathrm{~kW} \mathrm{~cm}^{-2}$ (c). Panels (d-f) show the corresponding derivative of the images in $(a-c)$ in order to enhance the border grains between the silver particles. g) Comparison of the size distribution of the features in the surface calculated from $(d-f)$. h) Comparison of the 2D-FFT for the images in $(\mathrm{a}-\mathrm{c})$.

less intense. Under $y$-polarization (Figure 3e), the transverse absorption band remains relatively narrow after laser treatment but shifts through the visible spectrum as a function of the laser power (Figure $3 \mathrm{f}$ ). Treatment at $P=3.5 \mathrm{~kW} \mathrm{~cm}^{-2}$ results in a red-shift from 650 to $750 \mathrm{~nm}$, while further increasing the laser power gradually shifts the band towards shorter wavelengths (a blue-shift up to $400 \mathrm{~nm}$ ). Blue-shifts are often attributed in the literature to a rounding of the nanoparticles or to a weakening of the electromagnetic coupling between them. ${ }^{[10,11]}$

Also noticeable in the $x$-polarized spectra is the enhancement of an additional absorption band at around $400 \mathrm{~nm}$ (Figure 3d) that is already present before treatment.

To interpret the evolution of plasmon absorption bands as a function of the laser power, the optical extinction coefficient of the system was modeled within the dipole approximation. Details are given in Figure S4 of the anisotropic effective medium modeling ${ }^{[27,28]}$ section of the Supporting Information. Despite the simplicity of the model and the crude approximation of the stripe morphologies (Figure S4a, Supporting Information), the simulations in Figure S4b,c reproduce qualitatively the measured evolution of the longitudinal and transverse plasmon resonances upon both low and high power laser treatments. Indeed, the broadening and red-shift of the longitudinal absorption band after low power treatment are well-reproduced by assuming a transition from densely packed nanoparticles to continuous stripes (Figure S4b). Meanwhile, the reversal of the transverse absorption band position and the loss of intensity of the longitudinal plasmon after high-power treatments are reproduced by assuming narrower stripes with a higher aspect ratio (Figure S4c).

Laser treatment of metals at low or moderate powers is generally known to affect their nanostructure through ablation mechanisms $^{[29]}$ or by heating effects, producing an enhanced atomic surface diffusion or even volume melting. ${ }^{[23]}$ In our experiment a significant ablation of silver can be discarded because no removal of silver was observed, as estimated from the height of stripes and the surface coverage. For ns-laser annealing with pulse durations longer than the metal electron-phonon relaxation time, ${ }^{[23,30]}$ heat accumulates in the metal. For continuous metal thin films deposited on $\mathrm{SiO}_{2} / \mathrm{Si}$ such localized annealing leads to melting and dewetting of the metal. ${ }^{[31,32]}$ Since the conditions of our experiment (i.e., around 10 pulses with an average power density of $\approx 7 \mathrm{~kW} \mathrm{~cm}{ }^{-2}$ per pulse for the treatment at maximum power reaching a particular zone of the sample surface) are comparable to those used to partially dewet $\mathrm{Ag}$ thin films from $\mathrm{SiO}_{2} / \mathrm{Si}^{[31,32]}$ we propose a two step mechanism to account for the structural evolution of the stripes upon laser treatment. Firstly, the metal nanostructures accumulate heat up to their melting temperature. Because of their small size and the densely packed state in the original films, they melt rapidly into Ag droplets, which according to literature 

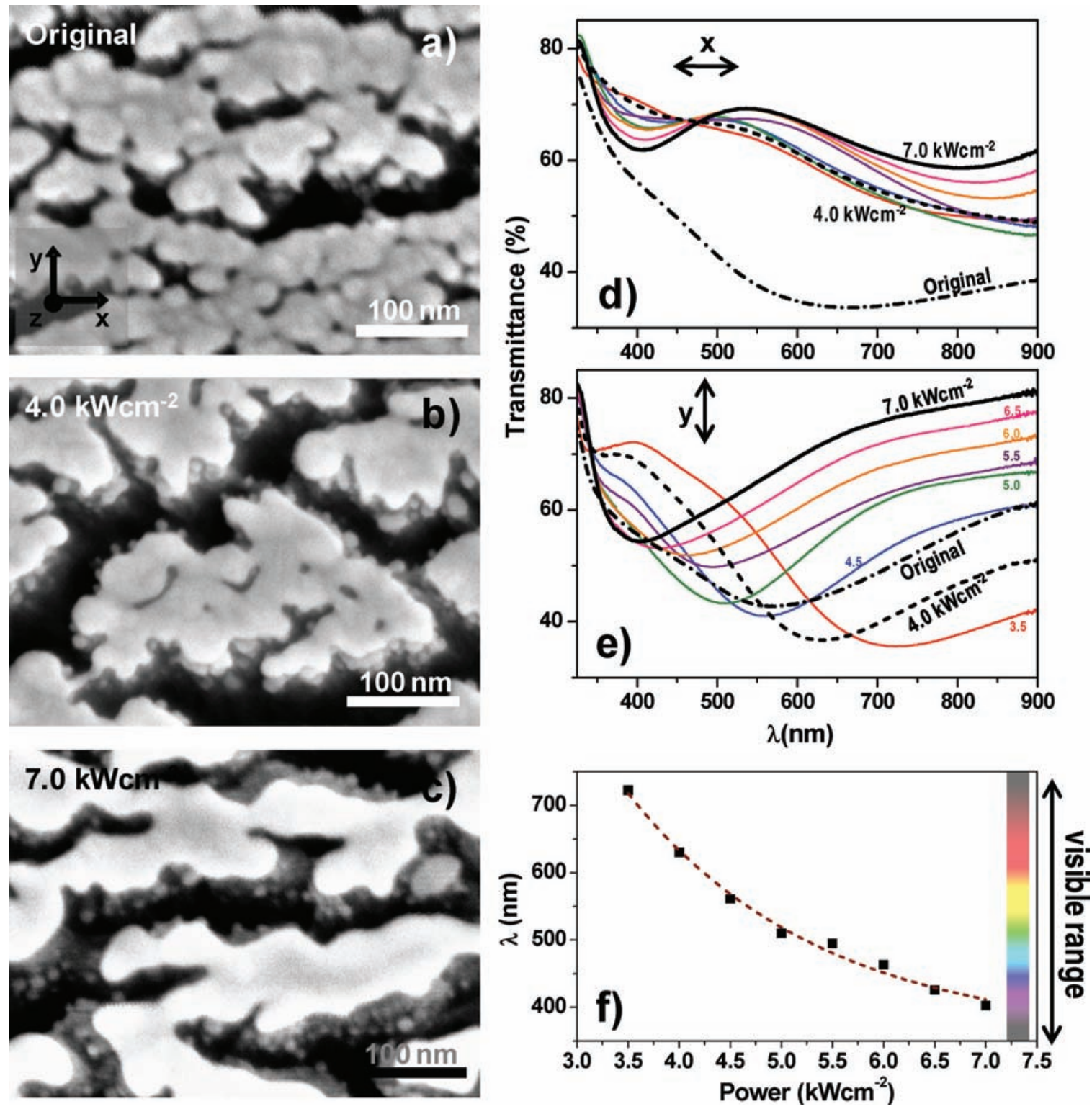

Figure 3. Left) $\mathrm{SEM}$ images of $\mathrm{AgNPs} / \mathrm{SiO}_{2} \mathrm{NCs}$ a) before laser treatment, b) treated at $4.0 \mathrm{~kW} \mathrm{~cm}^{-2}$, and c) treated at $7.0 \mathrm{~kW} \mathrm{~cm}^{-2}$. Right) Transmittance spectra measured before and after irradiation at different laser powers, as labeled for the incoming electric field oriented along d) the $x$-direction and e) the $\gamma$-direction. The color code is the same in both graphs. Spectra of the original sample and those treated at $4.0 \mathrm{~kW} \mathrm{~cm}{ }^{-2}$ and $7.0 \mathrm{~kW} \mathrm{~cm}-2$ have been highlighted to facilitate the comparison with the corresponding SEM images (see also Figure S3, Supporting Information). $\mathrm{f}$ ) Evolution of the spectral position of the transverse plasmon band as a function of laser output power.

do not tend to wet the silica substrate. ${ }^{[33]}$ Secondly, these droplets coalesce in the form of continuous and flat stripes formed along the bundle structure of the $\mathrm{SiO}_{2}$ with $\mathrm{NCs}$ acting as a template. ${ }^{[34]}$ Following the laser shot, the Ag NPs cool down, recrystallize, and freeze producing an interconnected droplet shape structure as shown by the SEM pictures on Figure 1b. As the laser power increases, the stripes undergo a partial dewetting. In all cases a residual set of small Ag NPs remain attached to the lateral faces of the $\mathrm{SiO}_{2} \mathrm{NCs}$ (see Figure 1 and Figures S2 and $\mathrm{S} 3$ in the Supporting Information).

Among the possible applications of the laser writing technique developed here, we would like to highlight its possibilities for optical patterning and coding. Figure 4a shows a series of colored samples obtained by ns-laser treatment of $\mathrm{AgNPs} / \mathrm{SiO}_{2} \mathrm{NCs}$ deposited on fused silica substrates. The pictures were acquired for samples illuminated with white light through a linear polarizer (Figure $4 \mathrm{~b}$ ). Upon azimuthal rotation of the samples, the illumination beam is toggled from $x$-polarized to $\gamma$-polarized and polarization modulated colors are then observed. In other words, they display an optical dichroism that can be tuned by varying the laser power. While $x$-polarized excitation brings out a monochromatic image (grey-blue tones) that is little affected by the laser power, a transition from a blue to a yellow color 

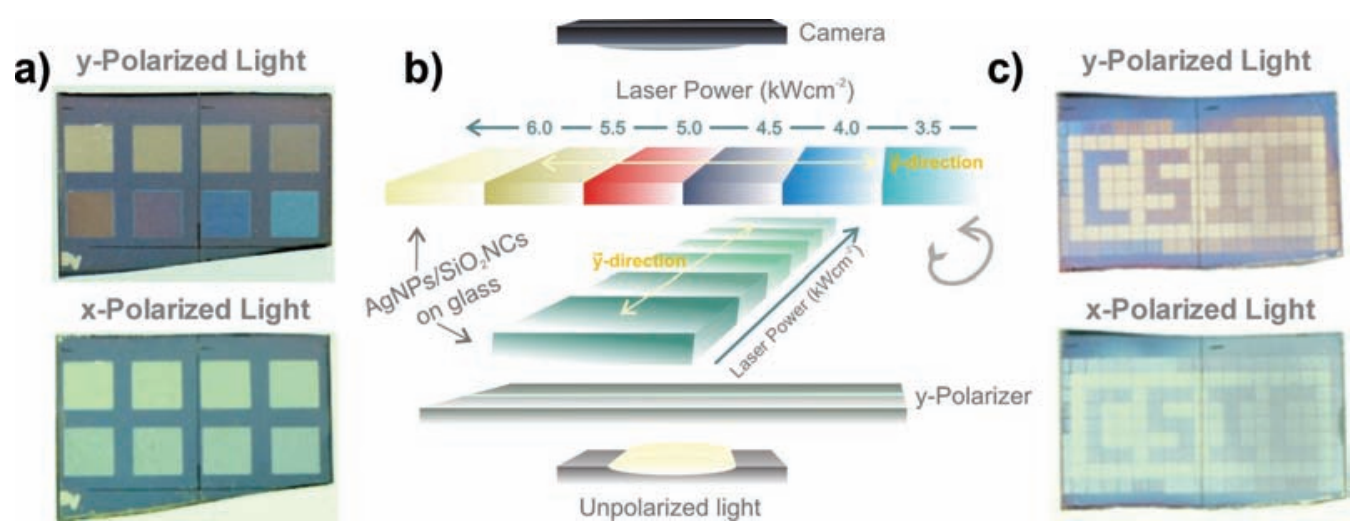

Figure 4. a) Pictures of $\mathrm{a} \approx 3.5 \times 2 \mathrm{~cm}^{2}$ glass slide coated with $\mathrm{AgNPs} / \mathrm{SiO}_{2} \mathrm{NCs}$ and subsequently treated by ns-laser. Patterning on the glass slide was achieved applying different laser po wer in each square zone. The relation between the apparent color of the sample and the percentage of the laser power is indicated in the sketch of the picture acquisition procedure (b). Under $x$-polarization, the pixel color remains monochrome, whereas a full-color transition as a function of the laser power is seen under $y$-polarization. c) Example of dichroic character encryption on glass slide coated with AgNPs/ $\mathrm{SiO}_{2} \mathrm{NCs}$. The size of the pixels is $\approx 0.20 \mathrm{~cm}$, but it can be decreased to the lateral resolution of the laser $(\approx 100 \mu \mathrm{m})$.

depending on laser power is seen under $\gamma$-polarized illumination. Optical patterning with lateral resolution at the scale of the laser writing is a clear application of this procedure. An illustration at the macroscale is shown in Figure $4 \mathrm{c}$ where the letters "CSIC" were formed by tiling a series of square plates subjected to laser irradiation with different powers. Beyond this polarization-dependent coloring of patterns at the macro- and microscales, the materials and procedure developed here might be used for optical encryption by writing characters that could only be read with a given polarization of the incoming light. Unfortunately the letters "CSIC" can be distinguished regardless of the polarization (Figure 4c). This behavior results from the weak dependence on laser power of the $x$-polarized transmittance of the films (Figure 3d). However, in view of data encryption applications, one can note in Figure 3d,e that the film transmittance at $490 \mathrm{~nm}$ is independent (strongly dependent) on laser power when measured under $x$-polarization ( $\gamma$-polarization). Successful dichroic encryption might thus be obtained by depositing the $\mathrm{AgNPs} / \mathrm{SiO}_{2} \mathrm{NCs}$ films onto a photonic structure acting as a 490-nm bandpass filter. Main advantages of this procedure with regard to other lithographic or more conventional laserbased writing procedures rely on its easy implementation (i.e., electron, ion, or resist lithography are clearly more complicated methods) and the fact that encryption and encoding efficiency will not only depend on the size of the written patterns but also on the need of a given polarized light to read the information.

In this article, we have demonstrated that irradiation of $\mathrm{Ag}$ NPs deposited onto bundled $\mathrm{SiO}_{2}$ nanocolumns with a commercial writing ns-laser enables a precise control of their optical dichroism, which is attributed to the formation of long range ordered silver nanostripes formed through 1D metal percolation and partial dewetting along and perpendicular to the bundling direction of the $\mathrm{SiO}_{2} \mathrm{NCs}$, respectively. We foresee that these results are a first step towards the use of AgNPs/ $\mathrm{SiO}_{2} \mathrm{NCs}$ for optical nanopatterning, encryption, and data storage applications.

\section{Experimental Section}

$\mathrm{SiO}_{2}$ thin films with tilted nanocolumns were grown by GLAD by placing the substrates at a glancing angle of $70^{\circ}$ with respect to the evaporator. $\mathrm{Ag}$ was deposited at room temperature onto the $\mathrm{SiO}_{2} \mathrm{NCs}$ by $\mathrm{DC}$ sputtering at $400 \mathrm{~V}$ in an $\operatorname{Ar}(2 \mathrm{mbar})$ atmosphere. Laser post-treatment was performed at room temperature with a $20 \mathrm{~W}$ diode-pumped Nd:YAC (Powerline E, Rofin-Baasel Inc.) unpolarized laser emitting at $1064 \mathrm{~nm}$ with an $\approx 100 \mathrm{~ns}$ pulse width and a $20 \mathrm{kHz}$ repetition rate. ${ }^{[35]}$ The samples were scanned with an $\approx 0.5 \mathrm{~mm}$ spot at $1 \mathrm{~m} \mathrm{~s}^{-1}$ speed. AgNPs/ $\mathrm{SiO}_{2} \mathrm{NCs}$ nanostructure was characterized by SEM with a Hitachi S5200 microscope. Optical transmittance of the films was measured with a Cary 100 spectrophotometer at normal incidence and in the 300-900 nm range with a 1-nm monochromator step. Noncontact AFM characterization was carried out with a Cervantes AFM system from NANOTEC and the 2D-FFT and further analyses of the images performed with the WSXM software. ${ }^{[36]}$

\section{Supporting Information}

Supporting Information is available from the Wiley Online Library or from the author.

\section{Acknowledgements}

Authors thank the Projects FUNCOAT CONSOLIDER-INGENIO CDS2008 - 0023, MAT2007 - 65764, MAT2010-18447, MAT2010-21228, CEN2007 - 2014, P09-TEP-5283, Domingo Martinez Foundation, and Juan de la Cierva Grant No JCl-2009 - 05098 for J.T.

[1] a) U. Kreibig, M. Volmer, Optical properties of metal clusters, Springer, Berlin, Germany 1995; b) W. A. Murray, W. L. Barnes, Adv. Mater. 2007, 19, 3771.

[2] H. Ibn El Ahrach, R. Bachelot, A. Vial, G. Lérondel, J. Plain, P. Royer, O. Soppera, Phys. Rev. Lett. 2007, 98, 107402.

[3] a) E. Hutter, J. H. Fendler, Adv. Mater. 2004, 16, 1685; b) J. Toudert, H. Fernandez, D. Babonneau, S. Camelio, T. Girardeau, J. Solis, Nanotechnology 2009, 20, 475705. 
[4] a) H. Y. Liang, Z. P. Li, W. Z. Wang, Y. S. Wu, H. X. Xu, Adv. Mater. 2009, 21, 4614; b) A. Biswas, I. S. Bayer, D. H. Dahanayaka, L. A. Bumm, Z. Li, F. Watanabe, R. Sharma, Y. Xu, A. S. Biris, M. G. Norton, E. Suhir, Nanotechnology 2009, 20, 325705.

[5] H. Hövel, S. Fritz, A. Hilger, U. Kreibig, M. Vollmer, Phys. Rev. B: Condens. Matter Mater. Phys. 1993, 48, 18178.

[6] J. Gonzalo, R. Serna, J. Solis, D. Babonneau, C. N. Afonso, J. Phys.: Condens. Matter 2003, 15, 3001.

[7] J. S. Biteen, L. A. Sweatlock, H. Mertens, N. S. Lewis, A. Polman, H. A. Atwater, J. Phys. Chem. C 2007, 111, 13372.

[8] J. P. Barnes, A. K. Petford-Long, R. C. Doole, R. Serna, J. Gonzalo, A. Suárez-Garcia, C. N. Afonso, D. Hole, Nanotechnology 2002, 13, 465.

[9] B. Palpant, B. Prevel, J. lerme, E. Cottancin, M. Pellarin, M. Treilleux, A. Perez, J. I. Vialle, M. Broyer, Phys. Rev. B: Condens. Matter Mater. Phys. 1998, 57, 1963.

[10] F. Stietz, Appl. Phys. A 2001, 72, 381.

[11] J. Toudert, S. Camelio, D. Babonneau, M. F. Denanot, T. Girardeau, J.P. Espinós, P. Yubero, A. R. Gonzalez-Elipe, J. Appl. Phys. 2005, 98, 114316.

[12] L. Simonot, D. Babonneau, S. Camelio, D. Lantiat, P. Guérin, B. Lamongie, V. Antad, Thin Solid Films 2010, 518, 2637.

[13] a) G. B. Smith, S. Dligatch, R. Sullivan, M. G. Hutchins, Solar Energy 1998, 62, 229; b) M. Suzuki, Y. Taga, J. Appl. Phys. 1992, 71, 2848.

[14] S. Camelio, D. Babonneau, D. Lantiat, L. Simonot, F. Pailloux, Phys. Rev. B: Condens. Matter Mater. Phys. 2009, 80, 155434.

[15] E. Fort, C. Ricolleau, J. Sau-Pueyo, Nano Lett. 2003, 3, 65.

[16] M. Suzuki, W. Maekita, K. Kishimoto, S. Teramura, K. Nakajima, K. Kimura, Y. Taga, J. Appl. Phys. 2005, 44, L193.

[17] J. R. Sánchez-Valencia, J. Toudert, A. Borras, C. Lopez-Santos, I. Ortega-Feliu, A. Barranco, A. R. Gonzalez-Elipe, Plasmonics 2010, $5,241$.

[18] H. Mertens, A. Polman, Appl. Phys. Lett. 2006, 89, 211107.

[19] M. Cavallini, C. Albonetti, F. Biscarini, Adv. Mater. 2009, 21, 1043.
[20] P. Greco, M. Cavallini, P. Stoliar, S. D. Quiroga, S. Dutta, S. Zacchini, M. C. Iapalucci, V. Morandi, S. Milita, P. G. Merli, F. Biscarini, J. Am. Chem. Soc. 2008, 130, 1043

[21] T. Wenzel, J. Bosbach, A. Goldmann, F. Stietz, F. Träger, Appl. Phys. B 1999, 69, 513.

[22] M. Kaempfe, H. Graener, A. Kiesow, A. Heilmann, Appl. Phys. Lett. 2001, 79, 1876.

[23] S. Link, M. El-Sayed, J. Phys. Chem. B 1999, 103, 8410.

[24] P. Zijlstra, J. W. M. Chon, M. Gu, Nature 2009, 409, 410.

[25] a) H. Van Kranenburg, C. Lodder, Mater. Sci. Eng. 1994, R11, 295; b) J. R. Sanchez-Valencia, J. Toudert, L. Gonzalez-Garcia, A. R. Gonzalez-Elipe, A. Barranco, Chem. Commun. 2010, 46, 4372.

[26] a) J. R. Sanchez-Valencia, A. Borras, A. Barranco, V. Rico, J. P. Espinos, A. R. Gonzalez-Elipe, Langmuir 2007, 24, 9460; b) V. Rico, A. Borras, F. Yubero, J. P. Espinos, F. Frutos, A. R. Gonzalez-Elipe, J. Phys. Chem. C 2009, 113, 3775.

[27] E. D. Palik, Handbook of Optical Constants, Academic Press, New York, USA 1985.

[28] A. Dakka, J. Lafait, C. Sella, S. Berthier, M. Abd-Lefdil, J. C. Martin, M. Maaza, Appl. Opt. 2000, 39, 2745

[29] W. Hoheisel, U. Schulte, M. Vollmer, R. Weidenauer, F. Träger, Appl. Surf. Sci. 1989, 36, 664

[30] S. J. Henley, J. D. Carey, S. R. P. Silva, Phys. Rev. B: Condens. Matter Mater. Phys. 2005, 72, 195408.

[31] J. Trice, D. Thomas, C. Favazza, R. Sureshkumar, R. Kalyanaraman, Phys. Rev. B: Condens. Matter Mater. Phys. 2007, 75, 235439.

[32] H. Krishna, N. Shirato, C. Favazza, R. Kalyanaraman, Phys. Chem. Chem. Phys. 2009, 11, 8136 .

[33] K. S. Gadre, T. L. Alford, J. Appl. Phys. 2003, 93, 919.

[34] F. A. Nichols, J. Appl. Phys. 1966, 37, 2805.

[35] A. Castelo, D. Nieto, C. Bao, M. T. Flores-Arias, M. V. Perez, C. Gomez-Reino, C. Lopez-Gascon, G. F. de la Fuente, Opt. Commun. 2007, 273, 193.

[36] I. Horcas, R. Fernandez, J. M. Gomez-Rodriguez, J. Colchero, J. Gomez-Herrero, A. M. Baro, Rev. Sci. Instrum. 2007, 78, 013705. 